\title{
Maternal Cardiovascular Hemodynamics in Normal and Preeclamptic Pregnancies Using Echocardiography
}

\author{
Sonali S Somani ${ }^{1 *}$, Sunita Sudhir ${ }^{2}$, Shashikant G Somani ${ }^{3}$ and Meerajuddin $\mathrm{S}^{4}$ \\ ${ }_{1,2}^{1,2}$ ssistant Professor Department of Obstetrics \& Gynecology, Kamineni Institute of Medical Sciences, India \\ ${ }^{3}$ Assistant Professor Department of Physiology, Kamineni Institute of Medical Sciences, India \\ ${ }^{4}$ Assistant Professor Department of Anaesthesiology, Kamineni Institute of Medical Sciences, India
}

*Corresponding author: Sonali Shashikant Somani, Aditya Arcade, Sri Sai Block C, Flat No 303, VST Signal, Nallakunta, Hyderabad, 500020 Telangana, India.

Submission: 眥 December 14, 2017; Published: 眥 April 19, 2018

\begin{abstract}
Background: Preeclampsia is associated with higher prevalence of abnormal global left ventricular function. So, present study was designed to compare these changes in normal and preeclamptic pregnancies using echocardiography.

Methods: In present study, 25 patients with pre-eclampsia with singleton pregnancy between 20-30 years (study group) were compared with 25 age matched healthy pregnant women (control group). They were non-invasively analyzed for cardiovascular hemodynamics using echocardiography. The data was analyzed using student's t- test. P value $<0.05$ was considered to be significant. Results: Mean stroke volume in pre-eclampsia group was $72.4 \pm 5.1 \mathrm{ml} v / \mathrm{s} 67.7 \pm 5.6 \mathrm{ml}$ in control group and the difference was statistically significant. Mean Cardiac output in preeclampsia group was64.2 $\pm 3.4 \mathrm{lit} / \mathrm{min} \mathrm{v} / \mathrm{s} 5.69 \pm 0.47 \mathrm{lit} / \mathrm{min}$ in control group which is statistically significant. Mean Systemic vascular resistance (dyne.sec cm-5) in pre-eclampsia group was $1468.22 \pm 5.7 \mathrm{v} / \mathrm{s} 957.7 \pm 131$ in control group which is statistically significant. Mean Ejection fraction (\%) in pre-eclampsia group was $68.31 \pm 5.1 \mathrm{v} / \mathrm{s} 66.54 \pm 4.7$ in control group which is statistically significant.
\end{abstract}

Conclusions: Doppler echocardiography provides an excellent non-invasive method for evaluation of hemodynamic changes during pregnancy. Therefore if it is introduced into antenatal protocol, could help to identify subset of pre-eclamptic women who are at high risk to developing complications and thereby can do early intervention. Key words: Echocardiography, Hemodynamics, Hypertension, Pregnancy.

\section{Introduction}

Pregnancy is a physiological phenomenon. It produces both anatomical and physiological changes in all the organs including reproductive organs, cardiovascular system, hemopoietic system, respiratory system and excretory system etc. It is associated with volume overload producing significant vascular and hemodynamic adaptations in cardiovascular physiology [1].They are necessary for successful pregnancy, but they impose further load on the heart. Preeclampsia is a multisystem disorder that occurs after 20 wks of pregnancy. Acute preeclampsia is associated with significantly higher prevalence of asymptomatic abnormal global left ventricular (LV) abnormal function/geometry and myocardial injury [2]. It is also associated with significantly higher risk of subsequent heart failure, ischemic and hypertensive heart diseases and related mortality in later life $[3,4]$. Many studies of women with preeclampsia have revealed diverse hemodynamic findings such as elevated cardiac output (CO), high vascular resistance, and reduced $\mathrm{CO}$ and myocardial contractility [5]. Impairment of LV diastolic function as well as systolic function appear very early in the course of heart disease [6]. Early detection and its treatment at an asymptomatic phase can help in improving the prognosis. In earlier days, gas exchange techniques were used to determine cardiac output. With development of cardiac catheterization, it was used to study right heart hemodynamics. The advent of echocardiography enabled serial noninvasive cardiovascular monitoring throughout pregnancy. Initially, investigators used M-mode but perhaps due to its limitations, results were not uniform [7]. Recently, Doppler echocardiography has been used which is reproducible and noninvasive technique suitable for pregnant Women [8].

Therefore echocardiography if introduced into the routine antenatal protocol, could help to identify women who are at high risk to develop cardiovascular complications and there by early intervention.

\section{Methods}

Present study was conducted at Department of Obstetrics and Gynecology in Kamineni Institute of Medical Sciences, Narketpally 
Nalgonda District, Telangana. It was prospective case controlled study conducted in 50 subjects during September 2015 to August 2016 over a period of 12 months. In present study, 25 patients with pre-eclampsia with singleton pregnancy between 20-30 years (study group) were compared with 25age matched healthy pregnant women (control group). Patients who refused to participate; those suffering from cardiovascular disease, diabetes mellitus, thyroid disorder, anaemia and multiple gestations were excluded. All subjects were informed in detail about aim and procedure of study; and written consent was taken for conduct of study.

A detailed obstetric history and thorough examination was done in all subjects. Age, Height, Weight were noted. Blood pressure was measured by sphygmomanometer in right arm in left lateral position after 10 minutes of rest by auscultator method. Onset of tapping sound was taken as systolic and muffling of Korotkoff sound was taken as diastolic blood pressure. An obstetric ultrasound scan was performed to confirm singleton pregnancy and gestational age. All subjects were subjected to echocardiography using Philips ANVISER-C model. Standard parasternal two dimensional long axis images were recorded and left ventricular diameters were measured in M mode (American Society of Echocardiography guidelines) [9]. Doppler Echocardiography was used to calculate stroke volume [10]. Simultaneously heart rate per minute was recorded. Cardiac output, Systemic vascular resistance and ejection fraction were calculated.

\section{Statistical Analysis}

The data was expressed as Mean \pm S.D and was analyzed using student's t-test P-value $<0.05$ was considered to be significant.

\section{Result}

(Table1-3)

Table 1: Comparison of mean age, mean height and mean weight of control and study groups $(\mathrm{n}=50)$.

\begin{tabular}{|c|c|c|c|}
\hline & Control Group $(n=25)$ & Study Group $(n=25)$ & P Value \\
\hline Mean age (years $\pm S D$ ) & $24.5 \pm 4.18$ & $25.91 \pm 3.24$ & $>0.05$ \\
\hline Mean height $(\mathrm{cm} \pm \mathrm{SD})$ & $154.34 \pm 2.78$ & $149.08 \pm 4.55$ & $>0.05$ \\
\hline Mean weight $\mathrm{Kg} \pm \mathrm{SD}$ ) & $55.4 \pm 3.04$ & $60.34 \pm 4.54$ & $<0.05$ \\
\hline
\end{tabular}

$P$ value: $<0.05$ - significant; Mean age and height in control and study groups were comparable.

Mean weight was more in study group which was statistically significant.

Table 2: Comparison of heart rate and blood pressure between study and control group ( $\mathrm{n}=50$ ) (Mean \pm SD). Hemodynamic parameters are more in study group then control group and are statistically significant.

\begin{tabular}{|c|c|c|c|}
\hline Parameter & Control Group (n=25) & Study Group(n=25) & P Value \\
\hline Heart rate (Beats/min) & $97.6 \pm 4.1$ & $106 \pm 8.05$ & $<0.05$ \\
\hline Systolic blood pressure (mm Hg) & $107.32 \pm 6.9$ & $114 \pm 8.4$ & $<0.05$ \\
\hline Diastolic blood pressure (mm Hg) & $71.5 \pm 5.1$ & $82 \pm 7.2$ & $<0.05$ \\
\hline
\end{tabular}

Table 3: Comparison of echocardiographic parameters between study and control group ( $n=50)($ Mean $\pm S D)$.

\begin{tabular}{|c|c|c|c|}
\hline Parameter & Control Group (n=25) & Study Group (n=25) & P Value \\
\hline Stroke volume (ml) & $67.7 \pm 5.6$ & $72.4 \pm 5.1$ & $<0.05$ \\
\hline Cardiac output (lit/min) & $5.69 \pm 0.47$ & $64.2 \pm 3.4$ & $<0.05$ \\
\hline $\begin{array}{c}\text { Systemic vascular resistance (dyne. } \\
\text { sec cm-5) }\end{array}$ & $957.7 \pm 131$ & $1468.22 \pm 5.7$ & $<0.05$ \\
\hline Ejection fraction (\%) & $66.54 \pm 4.7$ & $68.31 \pm 5.1$ & $<0.05$ \\
\hline
\end{tabular}

\section{Discussion}

Pregnancy is a condition of primary peripheral arterial vasodilatation, associated with hyperdynamic circulation with high CO and low SVR [11]. Preeclampsia is a disease unique to pregnancy that contributes substantially to maternal and fetal morbidity and mortality, and is associated with increased vascular resistance and hypertension [12]. Hypertension produces structural changes in the left ventricle usually accompanied by functional alterations and in majority of cases; these alterations precede clinical manifestations [13]. In normal pregnancy, an increased preload and a decreased after load favor an improved emptying of the left ventricle during systole and a reduction of the end-systolic pressure [14]. In preeclamptic women, the elevated after load is linked with a reduced emptying of the left ventricle and elevated end-systolic pressure. Rizwana Solanki et al found a statistically significant difference in mean SV between pre eclamptics and normotensive women groups' studied [5].

Results of present study were also comparable to study done by Dennis et al. [15]. They observed cardiac output was higher in PIH group as compared to the controls. They stated that cardiac output is increased in women with untreated pre eclampsia due to an increase in stroke volume. Solanki et al. [4]. Found cardiac output was significantly higher in pre eclamptic patients5 our results were comparable to their. In present study, systemic vascular resistance was more in pre eclimptic patients. This is in accordance with study of Solanki et al. [5]. 


\section{Conclusion}

Doppler echocardiography provides an excellent non-invasive method for the evaluation and serial analysis of hemodynamic changes during pregnancy. They help in distinguishing abnormal changes from maternal physiologic changes. Preeclampsia still contributes to a majority of maternal mortality and morbidity. Therefore echocardiography if introduced into the antenatal protocol, could help to identify subset of preeclamptic women who are at high risk to develop cardiovascular complications and thereby can do early intervention.

\section{Limitation}

It was performed in a single hospital therefore sample may not be representative of all Indian women. Therefore we recommend a cross-sectional multicentric study to confirm results.

\section{Future}

Further studies can be made by 3D echocardiography which is based on direct volumetric quantification, which is independent of geometric assumption of Left Ventricle

\section{Acknowledgement}

We express gratitude to all patients. They are grateful to authors, editors and publishers of all those articles, journals from where literature for this article has been reviewed and discussed.

\section{References}

1. Sonali S Somani, Sunandini R, Shashikant G Somani (2016) Role of echocardiography for assessment of cardiovascular haemodynamics during pregnancy. Int J Reprod Contracept Obstet Gynecol 5(1): 84-89.

2. Melchiorre K, Sutherland GR, Baltabaeva A, Liberati M, Thilaganathan B (2011) Maternal cardiac dysfunction and remodeling in women with preeclampsia at term. Hypertension 57(1): 85-93.

3. Lykke JA, Langhoff Roos J, Sibai BM, Funai EF, Triche EW, et al. (2009) Hypertensive pregnancy disorders and subsequent cardiovascular morbidity and type 2 diabetes mellitus in the mother. Hypertension 53(6): 944-951.

4. Mongraw Chaffin ML, Cirillo PM, Cohn BA (2010) Preeclampsia and cardiovascular disease death: prospective evidence from the child health and development studies cohort. Hypertension 56(1): 166-71.

5. Solanki R, Maitra N (2011) Echocardiographic assessment of cardiovascular hemodynamics in preeclampsia. J Obstet Gynaecol India 61(5): 519-522.

6. Kuznetsova T, Herbots L, Jin Y, Stolarz Skrzypek K, Staessen JA (2010) Systolic and diastolic left ventricular dysfunction: From risk factors to overt heart failure. Expert Rev Cardiovasc Ther 8(2): 251-258.

7. Vered Z, Poler SM, Gibson P, Wlody D, Wrez J (1991) Noninvasive Detection of the morphologic and hemodynamic changes during normal pregnancy. Clin Cardiol 14(4): 327-334.

8. Robson SC, Hunter S, Boys RJ, Dunlop W (19589) Serial study of factors influencing changes in cardiac output during human pregnancy. Am J Physiol 256(4 Pt 2): 1060-1065.

9. Sahn DJ, DeMaria A, Kisslo J, Weyman A (1978) Recommendations regarding quantitation in M-mode echocardiography: results of a survey of echocardiographic measurements. Circulation 58(6): 1072-1083.

10. Van Oppen AC, Stigter RH, Bruinse HW (1996) Cardiac output in normal pregnancy: A critical review. Obstet Gynecol 87(2): 310-318.

11. Schrier RW (1989) Pathogenesis of sodium and water retention in high-output and low-output cardiac failure, nephrotic syndrome and pregnancy (2). N Engl J Med 319(17): 1127-1134.

12. Easterling TR, Benedetti TJ, Schmucker BC, Millard SP (1990) Maternal hemodynamics in normal and preeclamptic pregnancies: A longitudinal study. Obstet Gynecol 76(6):1061-1069.

13. Escudero EM, Favaloro LE, Moreira C, Plastino JA, Pisano O (1988) Study of the left ventricular function in pregnancy-induced hypertension. Clin Cardiol 11(5): 329-333.

14. Valensise H, Novelli GP, Vasapollo B, Di Ruzza G, Romanini ME, et al. (2001) Maternal diastolic dysfunction and left ventricular geometry in gestational hypertension. Hypertension 37(5): 1209-1215.

15. Dennis AT, Castro S, Carrc C, Simmons S, Permezel M, et.al (2012) Haemodynamics in women with untreated preeclampsia. Anaesthesia 67(10): 1105-1118.

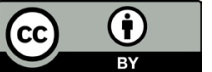

Creative Commons Attribution 4.0 International License

For possible submissions Click Here

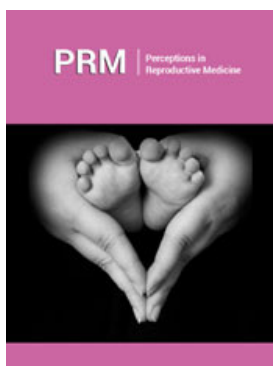

\section{Perceptions in Reproductive Medicine}

\section{Benefits of Publishing with us}

- High-level peer review and editorial services

- Freely accessible online immediately upon publication

- Authors retain the copyright to their work

- Licensing it under a Creative Commons license

- Visibility through different online platforms 